\section{Ascending pneumonia complicating endoscopic therapy of a pancreatic abscess}

A 71-year-old man with multiorgan failure caused by necrotizing pancreatitis was discharged from the intensive care unit after a 6-week stay. Two weeks later he developed a spiking fever, abdominal tenderness, and an abdominal mass. Computed tomography revealed a large pancreatic abscess that was compressing the duodenum and gastric wall ( $\bullet$ Fig. 1). Because of the interposition of bowel, transcutaneous drainage was risky and an endoscopic approach was chosen. After transmural duodenocystic puncture and dilation, a 7-Fr double-pigtail stent and a nasocystic tube were inserted ( $\bullet$ Fig. 2). Two days after the cystogastrostomy the patient developed dyspnea and hypoxia, became drowsy, and had to be intubated and mechanically ventilated. Chest radiography revealed patchy infiltrates in the lower lobe of the right lung and bronchoalveolar lavage was performed. Cultures grew Klebsiella oxytoca, Enterococcus faecium, and Candida glabrata, and these organisms were also grown from cultures of the aspirate from the pancreatic abscess. The patient died of septic multiorgan failure after a long period of intensive-care treatment.

Whereas early mortality in severe acute pancreatitis is due to the systemic inflammatory response syndrome, late mortality of pancreatitis is determined by the development of secondary infection of necrotic tissue or pseudocysts [1]. Infection of pseudocysts leads to pancreatic abscess, a common complication of severe acute pancreatitis with a mortality of around $9 \%$ [2].

Several case series have proposed an endoscopic approach for the treatment of both infected pancreatic necrosis and perigastric pancreatic abscesses. Success rates of $90 \%$ for complete resolution of infected necrosis or abscess have been reported $[3,4]$, although hemorrhage and perforation have been described as complications of these interventions [5].

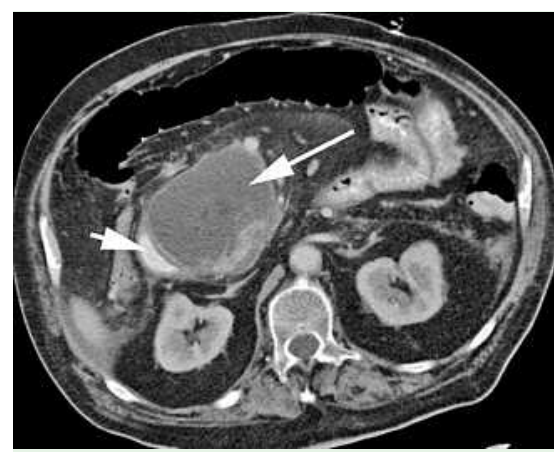

Fig. 1 Abdominal computed tomographic scan showing a large cystic structure (long arrow) compressing the duodenal lumen (short arrow).

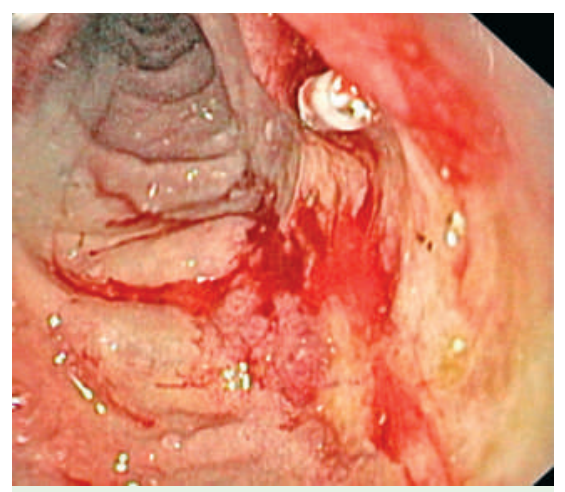

Fig. 2 Endoscopic view of the duodenum after duodenocystic puncture, with a stent in place.

The causal relationship linking a nosocomial pneumonia with an endoscopic procedure performed several days before can be difficult to confirm. In this case, however, the microbiological results for the cystic aspirate and the bronchoalveolar lavage effluent were identical, and the close temporal relationship between the endoscopic intervention and the pneumonia suggests that the pneumonia must be regarded as a complication of that intervention.

Endoscopy_UCTN_Code_CPL_1AK_2AG
A. Umgelter ${ }^{1}$, C. Prinz ${ }^{1}$, J. Gaa², W. Huber ${ }^{1}$

${ }^{1}$ II Medizinische Klinik und Poliklinik, Klinikum rechts der Isar der Technischen Universität München, München, Germany

2 Institut für Röntgendiagnostik, Klinikum rechts der Isar der Technischen Universität München, München, Germany

\section{References}

1 Neoptolemos JP, Raraty M, Finch $M$ et al. Acute pancreatitis: the substantial human and financial costs. Gut 1998; 42: 886-891

2 Mithöfer K, Mueller PR, Warshaw AL. Interventional and surgical treatment of pancreatic abscess. World J Surg 1997; 21: $162-$ 168

3 Park JJ, Kim SS, Koo YS et al. Definitive treatment of pancreatic abscess by endoscopic transmural drainage. Gastrointest Endosc 2002; 55: 256-262

4 Seewald S, Groth S, Omar S et al. Aggressive endoscopic therapy for pancreatic necrosis and pancreatic abscess: a new safe and effective treatment algorithm. Gastrointest Endosc 2005; 62: 92 - 100

5 Hookey LC, Debroux S, Delhaye M et al. Endoscopic drainage of pancreatic-fluid collections in 116 patients: a comparison of etiologies, drainage techniques, and outcomes. Gastrointest Endosc 2006; 63: 635-643

Bibliography

DOI $10.1055 / \mathrm{s}-2007-966606$

Endoscopy 2007; 39: E267

(c) Georg Thieme Verlag KG Stuttgart · New York . ISSN 0013-726X

Corresponding author

\section{A. Umgelter, MD}

II Medizinische Klinik und Poliklinik Klinikum rechts der Isar der Technischen Universität München Ismaningerstraße 22 81675 München

Germany

Fax: +49-89-41404808

andreas.umgelter@Irz.tu-muenchen.de 